will investigate dietary intervention as a potential disease-modifying factor. CONFLICT OF INTEREST DESCRIPTION: NA, nothing to disclose.

4183

Micro-consults: An effective tool for meeting statistical support needs in an academic medical research center Sandra Taylor ${ }^{1}$, and Susan L. Stewart ${ }^{1}$

${ }^{1}$ University of California, Davis

OBJECTIVES/GOALS: Access to biostatistics expertise is essential for a successful clinical and translational research program. However, demand for statistical support at academic research centers can strain the capacity of biostatistics units. Our objective was to efficiently increase access to statistical expertise. METHODS/ STUDY POPULATION: In cooperation with the Cancer Center Biostatistics Shared Resource, we replaced an informal 1-hour drop-in consultation program with structured office hours to provide statistical support to clinical and translational researchers at the University of California, Davis Medical Center. We doubled office hours to 2 hours per week and established six 20-minute appointments. Two Ph.D. level statisticians staff office hours. Researchers schedule appointments through Acuity Scheduling, a free on-line resource. Availability of the service is advertised monthly by sending an informational flyer to various university listservs. RESULTS/ANTICIPATED RESULTS: Prior to implementing the program in 2014, we averaged 91 office hour consults per year. Subsequently, consultations jumped to 171 in 2014 and have averaged 150 per year since then. Office hours attract students, residents, staff and faculty from a wide range of disciplines including the Schools of Medicine, Nursing, Veterinary Medicine and basic science departments. Project types span the clinical and translational spectrum covering lab, animal, clinical and population-level studies. Most consults related to data analysis and interpretation (57\%) followed by sample size calculations/study design (29\%) and response to reviewers $(4 \%)$, with general statistical advice as the remainder. DISCUSSION/SIGNIFICANCE OF IMPACT: With 6 micro-consults per week, we can meet with many investigators and triage their statistical support needs. This program has proved very popular and was highly rated in a recent user survey, with several investigators noting that the consults facilitated successful publications and proposals.

\section{Predictors of Reintubation After Cardiac Surgery}

Robert Edward Freundlich ${ }^{1}$, Gen $\mathrm{Li}^{1}$, Jonathan $\mathrm{P}$ Wanderer ${ }^{1}$, Frederic T Billings ${ }^{1}$, Henry Domenico ${ }^{1}$, Daniel Byrne ${ }^{1}$, and Pratik Pandharipande ${ }^{1}$

${ }^{1}$ Vanderbilt University Medical Center

OBJECTIVES/GOALS: We modeled risk of reintubation within 48 hours of cardiac surgery using variables available in the electronic health record (EHR). This model will guide recruitment for a prospective, pragmatic clinical trial entirely embedded within the EHR among those at high risk of reintubation. METHODS/ STUDY POPULATION: All adult patients admitted to the cardiac intensive care unit following cardiac surgery involving thoracotomy or sternotomy were eligible for inclusion. Data were obtained from operational and analytical databases integrated into the Epic EHR, as well as institutional and departmental-derived data warehouses, using structured query language. Variables were screened for inclusion in the model based on clinical relevance, availability in the EHR as structured data, and likelihood of timely documentation during routine clinical care, in the hopes of obtaining a maximally-pragmatic model. RESULTS/ANTICIPATED RESULTS: A total of 2325 patients met inclusion criteria between November 2, 2017 and November 2, 2019. Of these patients, $68.4 \%$ were male. Median age was 63.0. The primary outcome of reintubation occurred in $112 / 2325(4.8 \%)$ of patients within 48 hours and $177 / 2325(7.6 \%)$ at any point in the subsequent hospital encounter. Univariate screening and iterative model development revealed numerous strong candidate predictors (ANOVA plot, figure 1), resulting in a model with acceptable calibration (calibration plot, figure 2), $c=0.666$. DISCUSSION/SIGNIFICANCE OF IMPACT: Reintubation is common after cardiac surgery. Risk factors are available in the EHR. We are integrating this model into the EHR to support realtime risk estimation and to recruit and randomize high-risk patients into a clinical trial comparing post-extubation high flow nasal cannula with usual care. CONFLICT OF INTEREST DESCRIPTION: REF has received grant funding and consulting fees from Medtronic for research on inpatient monitoring.

4099

\section{Principles of Statistical Education for Translational Scientists in the Age of Rigor, Reproducibility, and Reporting}

Emilia Bagiella, $\mathrm{PhD}^{1}$, Paul Christos ${ }^{1}$, Mimi Kim, ScD², Shing Lee ${ }^{1}$, Roger Vaughan ${ }^{3}$, and Judy Zhong ${ }^{4}$

${ }^{1}$ Mount Sinai School of Medicine; ${ }^{2}$ Albert Einstein College of

Medicine; ${ }^{3}$ Rockefeller University; ${ }^{4}$ New York University General

Clinical Research Center

OBJECTIVES/GOALS: To describe principles, best practices, and techniques recommended to instill deep understanding of the application and interpretation of statistical techniques and statistical inference among translational scientists and trainees, that best support the concepts of scientific Rigor, Reproducibility and Reporting. METHODS/ STUDY POPULATION: Each of the six New York City Area Biostatistics, Epidemiology and Research Design (BERD) resources have strong educational programs, novel curricular components, and creative strategies, implemented by award winning educators. To capitalize on shared knowledge, innovation, and resources, the six teams formed the New York City Area BERD Collaborative (NYC-ABC) comprised of BERD resources from Mt. Sinai, Cornell, Einstein, Columbia, Rockefeller, and NYU. The collaborative suggests principles, concepts, tools and approaches to support the concepts of scientific Rigor, Reproducibility and Reporting in translational science. RESULTS/ ANTICIPATED RESULTS: Principles:

- Value of team science approach and including biostatisticians early and often.

- Carefully designing experiments to reduce bias and increase precision.

- Trainees' focus is often on "statistical significance" and the p-value. Consequences of data dredging/p-hacking, and the impact of sample size and other factors on statistical significance. 